\title{
Performance Evaluation of Block based Video Watermarking after Attacks
}

\author{
Garima Gupta, Vijay Kumar Gupta, \\ Mahesh Chandra, Savita Joshi
}

\begin{abstract}
In today's era use of digital media is most popular way of communication. Digital media covers images, videos and animations available online. The easy methods of accessing, copying and editing digital media have made them more popular. With several advantages these easy methods of copying and editing data have created some big issues like ownership identification. This increases the demand of protecting online digital media. Watermarking is solution of such problem. In this work, a block-based method has been proposed for video watermarking that uses a key at the time of embedding and extraction. Some frames are selected from the video according to a key. Watermark is embedded on the selected frames after dividing into parts called blocks. Each part of the watermark is embedded in one selected frame of the video. This method increases the security of the system as the complete watermark cannot be extracted without knowing the positions of watermarked frames and the position of the block in that frame. Watermarking is performed in the Discrete Wavelet Transform domain after scaling of watermark data. To show the authenticity of proposed scheme various attacks are applied on different watermarked video frames and extracted watermark results are shown under different tables.
\end{abstract}

Keywords--- Video Watermarking, DWT, SVD, Secret Key, Blocks of the Watermark, PSNR, NC, Attacks.

\section{INTRODUCTION} also. Watermarking is helpful for the security of online digital content. Watermark is embedded in the digital content before uploading it online. The watermark embedded should be imperceptible to the users. Also, if an attacker wants to crack the watermark it should prove to be robust(Rao \& Rao, 2016)(Tsai, Huang, \& Kuo, 2011)

Piracy seems a major problem for the film industry (Koch, Smith, \& Telang, 2011)( Stelter \& Stone, Feb. 2009.)The main reason is the use of camcorder inside theatures. Australian economy lost more than $\$ 1.37$ billion in revenue in a duration of one yeardue to movie theft (Economic consequences of movie piracy - Australia , 2011). So, concern of protecting the copyright of digital video content is increasing. (Piva, Bartolini, \& Barni, 2002)( $\mathrm{Lu} \&$ Liao, Multipurpose watermarking for image authentication and protection, 2001)( Lu, Huang, Sze, \& Lia, 2000).

There is large literature available on watermarking methods as this field is not new and already number of

Manuscript received September 16, 2019.

Garima Gupta, ECE Department, Inderprastha Engineering College, AKTU, Ghaziabad, Uttar Pradesh, India. (e-mail: garima1204@ gmail.com)

Vijay Kumar Gupta, ECE Department, Inderprastha Engineering College, AKTU, Ghaziabad, Uttar Pradesh, India. (e-mail: guptavk76@gmail.com)

Mahesh Chandra, ECE Department, Birla Institute of Technology, Mesra, Ranchi, India. (e-mail: shrotriya69@rediffmail.com)

Savita Joshi, ECE Department, Inderprastha Engineering College, AKTU, Ghaziabad,Uttar Pradesh, India. (e-mail: savitakjoshi@yahoo.com)
Fast growing digital world is giving rise to safety issues

methods have been given by researchers. (Asikuzzaman \& Pickering, 2017) reviewed all the methods. Watermarking methods based on transform domain are more robust when compared with spatial domain. Then modified transform domain methods of watermarking shows an improvement over simple transform methods (Allali, Elabbadi, \& Elhassa, 2012). SVD based watermarking shows improved results(Thind \& Jindal, 2015) (Wenhai Kong, Wu, \& Xiamu, 2006). Mostly all methods are concerned about the PSNR value of watermarked data when compared with the original one (Sharma \& Mann, 2017). This method of watermarking takes the advantage of DWT- SVD and block based methods of watermarking. Authenticity of the watermarking method may be calculated with the attack bearing capabilities of that scheme. Proposed block based approach of watermarking is tested against seven attacks and some parameters like PSNR and NC are used for performance calculation after these attacks.

\section{SVD DECOMPOSITION}

SVD is an orthogonal matrix decomposition method. It is robust, reliable and attractive algebraic transform for image processing. Any digital Image of size $\mathrm{M} \times \mathrm{N}$, with $\mathrm{M} \geq \mathrm{N}$, can be represented by its SVD.X $=\mathrm{U} \times \mathrm{S} \times \mathrm{V}^{\mathrm{T}}$

$\mathrm{U}(\mathrm{M} \times \mathrm{M})$ : $\mathrm{U}$ is orthogonal, $\mathrm{U}^{\mathrm{T}} \mathrm{U}=\mathrm{I}$; columns of $\mathrm{U}$ are the orthogonal eigenvectors of $X^{T} X$ and left singular vectors of $\mathrm{X} . \mathrm{V}(\mathrm{N} \times \mathrm{N})$ : $\mathrm{V}$ is orthogonal or $\mathrm{V}^{\mathrm{T}} \mathrm{V}=\mathrm{I}$; columns of $\mathrm{V}$ are the orthogonal eigenvectors of $\mathrm{X}^{\mathrm{T}} \mathrm{X}$ and right singular vectors of $\mathrm{X}$.

$S(\mathrm{M} \mathrm{x} \mathrm{N})$ : It is a diagonal matrix consisting ofnon-zero values in descending order called the singular values.

\section{WATERMARK EMBEDDING DESIGN}

The method used is based on DWT. Here input is acolored video and watermarking is performed as shown in Fig 1. Input video is converted into frames. Any frame is selected according to the secret key. Key is like a secret password known to the owner of the video, which is helpfulto select the frames. Preprocess the frame and apply DWT on subbandsLLh, LHh, HLh, HHh. Again apply DWT on the LHh and get 4 sub-bands again LL2, LH2, HL2, HH2. Convert LH2 into 9 blocks with the 3-row block and 3 column block. For the host, it is shown by gh.
$[\operatorname{gh}\{1,1\}$
$[\operatorname{gh}\{2,1\}$
$[\operatorname{gh}\{3,1\}$
$\operatorname{gh}\{1,2\}$
$\operatorname{gh}\{2,2\}$
$\operatorname{gh}\{3,2\}$
$\operatorname{gh}\{1,3\}]$
$\operatorname{gh}\{2,3\}]$
$\operatorname{gh}\{3,3\}]$

Now take the watermark image and do pre-process operations on it. 
Convert preprocessed watermark image to binary image. Convert the watermark image into 4 subbandsLLw, LHw, HLw, HHw. Select LLw and divide it into the 9 blocks as done for LHh. For watermark, it is represented by gw.

$\begin{array}{lll}{[\operatorname{gw}\{1,1\}} & \operatorname{gw}\{1,2\} & \operatorname{gw}\{1,3\}] \\ {[\operatorname{gw}\{2,1\}} & \operatorname{gw}\{2,2\} & \operatorname{gw}\{2,3\}] \\ {[\operatorname{gw}\{3,1\}} & \operatorname{gw}\{3,2\} & \operatorname{gw}\{3,3\}]\end{array}$

Select a block of the watermark and apply SVD on it to get U2, S2, V2. Here, all the 9 blocks have been selected one by one and watermarking is shown with each watermark block. Embedding is performed as $\mathrm{S}=\mathrm{S} 1+\mathrm{c} * \mathrm{~S} 2$. Here c is a scaling factor. To get back the watermarked image, apply IDWT two times. Watermarked video is made again with the watermarked frames.

\section{WATERMARK EXTRACTION}

Extraction of watermark is the process to get back embedded watermark from watermarked video. For this watermarked video is taken as input and locate the frames with the watermark.Select watermark frame and 2 times DWT is applied on the watermarked image to get extracted sub-bands -LLe3, LHe3, HLe3 and HHe3. Select LHe3, a watermark is embedded in this band and divide the watermarked band into 9 blocks. Find SVD of the located block and get [U3, S3, V3]. Locate that block where the watermark is present. All the nine block are selected one by one to extract the parts of watermark. From S3, extraction is performed with the following equation, $S w=(S 3-$ $S 1) / c$. Here $S w$ is the extracted watermark. Extracted watermark parts are represented in Table 1. The extracted watermark is compared with the original watermark for performance calculation. Process of watermark extraction is shown in Fig.2.

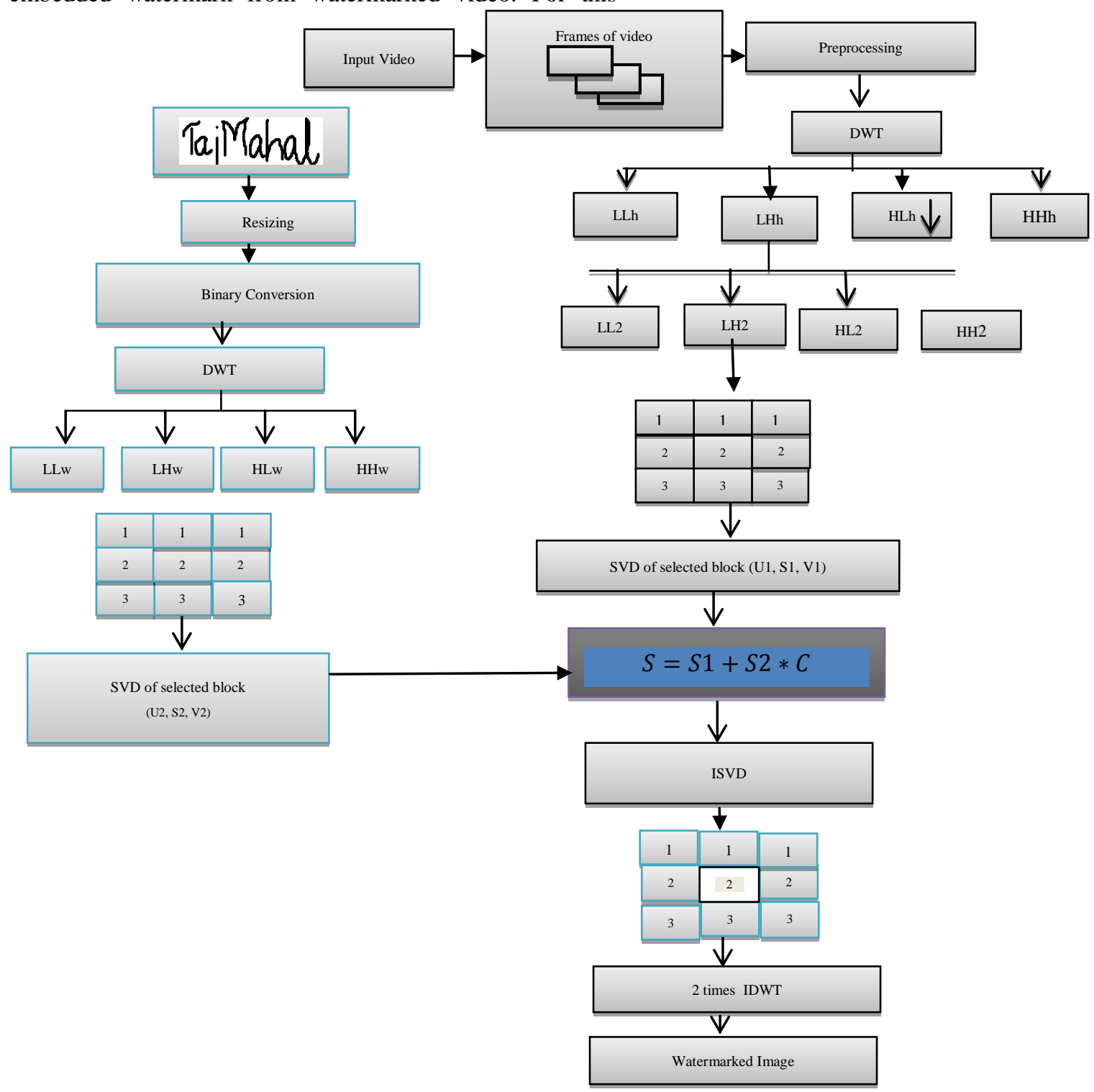

Fig. 1: Watermark Embedding Design 


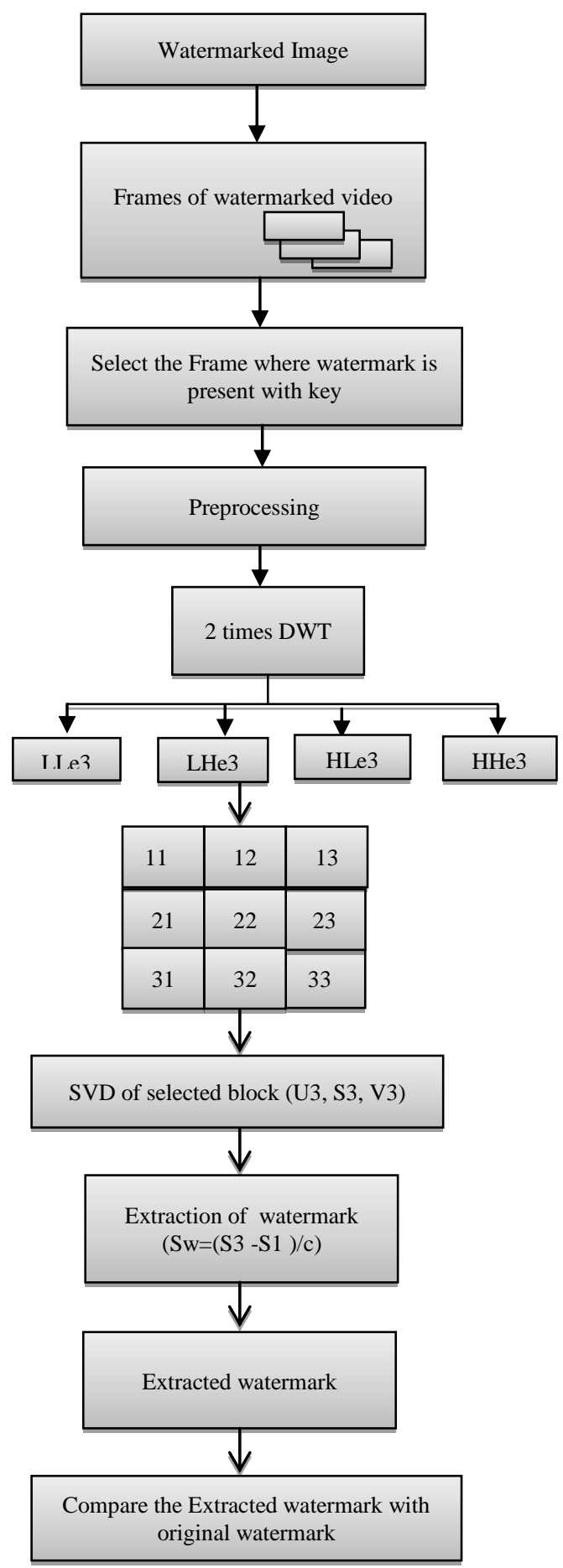

Fig. 2: Watermark Extraction Design

\section{EFFECT OF ATTACKSAFTER BLOCKWATERMARKING}

To check the authenticity of this scheme, a number of videos are taken. From these, results of 10 videos are shown in the tabular form. Videos that are taken are standard videos generally used in video processing. These are videos of Akiyo, Carphone, Claire, Coastguard, flower, foreman, mother-daughter, news, sports and wildlife. From these every entire $90^{\text {th }}$ frame has been selected. Watermarking is applied on this frame using block based DWT-SVD method. Watermarked frames of these videos are undergone through various attacks. (Kutter, Voloshynovskiy , \& Herrigel, 2000)given an overview about Watermark copy attack.(Kong, Yang, Wu, \& Niu, 2006) have shown attacks on watermarked frames. (Hsiao, Chen, Chien, \& Chen, 2007), (Khan, Tahir, Majid, \& Choi, 2008)(Peng, Wang, \& Wang, 2010) applied machine learning for extracting the watermark information after attacks in image watermarking. Attacks that are applied here are Gaussian attack, salt and pepper noise, speckle noise, Histogram equalization, cropping, 90 degree rotation, 180 degree rotation etc. After application of attacks watermark is extracted back from the attacked watermarked frames.

\section{Gaussian Noise Attack}

First attack applied is Gaussian noise attack. In this, the watermarked frame will change as if Gaussian noise is applied to it. Gaussian noise or normal noise is a random noise and effects both dark and light areas of an image. The Probability Density Function (PDF) of Gaussian noise is equal to that of the normal distribution, which is also known as the Gaussian distribution. The PDF of Gaussian random variable, $x$, is given by Eqn. (1).

$$
p(z)=\frac{1}{\sqrt{2 \pi \sigma}} e^{-(x-\bar{x})^{2} / 2 \sigma^{2}}
$$

Where,

$x$ represents intensity,

$\bar{x}$ shows mean value of $x$.

$\sigma$ is the standard deviation

$$
\sigma^{2} \text { is the variance of } x \text {. }
$$

\section{Salt and Pepper Noise}

This noise is also known as shot noise and bipolar impulse noise. This type of noise is caused by sudden disturbances in the images mainly due to sensor and memory problems. Salt is white dot and pepper is black dot on the image. These black and white dots have pixel values different as compared to background of that dot. These dots are removed by using median filtering. PDF of this noise is given by-

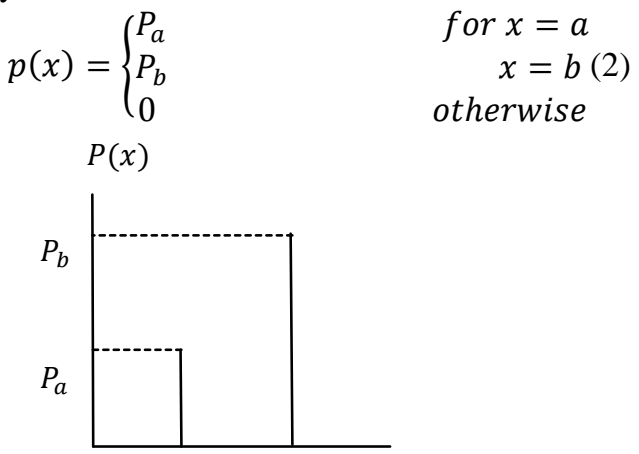

Figure 3: PDF of salt and Pepper noise

\section{Speckle Noise}

This noise is the inherent characteristic of ultrasound imaging. This noise is random and deterministic in an image.Table 4 shows the effect of Speckle noise. In this table extracted watermark after this attack, PSNR value and $\mathrm{NC}$ value is shown for all the 10 videos.

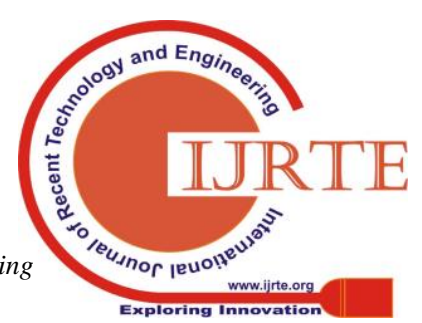




\section{Histogram Equalization}

Histogram method is used to uniformly distribute the intensity values. This enhances the quality of the image. This equalization is based on redistribution of the intensity values of images so that clarity of the image may be boosted. The effect of Histogram equalization is taken as an attack, as it changes the watermarked image. Effect on the extracted image after histogram equalization is shown in the Table 5.

\section{Cropping}

Cropping is to cut some part of image. This is generally done when one is interested in a particular area of the image. In cropping if the part of watermarked image where embedding is done, has been removed completely, then it is not possible to extract the watermark otherwise watermark can be extracted. Table 6 shows the effect of Cropping.

\section{Rotation $\left(90^{\circ}\right.$ and $\left.180^{\circ}\right)$}

In rotation also there is possibility that part of embedded watermark is moved at some other position, so some times it is somewhat difficult to extract watermark. Table 7 and Table 8 shows the effect of Rotation.

\section{VISUAL RESULTS}

An experiment has been performed on Akiyo video containing more than 300 frames. In this experiment, PSNR value has been found for watermarked when compared with the host image. Again Normalized correlation has been found in between the extracted watermark and original watermark as well as between watermarked image after the attack and the original host image. The experiment is performed on Matlab R2013a. Some frames have been selected according to the key. Table 1 shows the PSNR value, watermarked frame, extracted watermark and block with which extraction has been done. Here the value of scaling factor is 0.3 . Frame number in which watermark is embedded is known to the owner and will work as the key. As it can be seen that a part of the watermark has been extracted from each frame and to completely extract the watermark all frames need to be located that is only known to the owner. Here is video contains more than 300 frames. The watermark is divided into 9 parts.

Table 2, Table 3, Table 4, Table 5, Table 6, Table 7 and Table 8 shows the effect of Gaussian noise, salt and pepper noise, Speckle noise, Histogram equalization, Cropping, Rotation $90^{\circ}$, Rotation $180^{\circ}$ on the watermarked frame respectively.Videos taken are the standard videos like akiyo, carphone, Claire, Coastguard, Flower, Motherdaughter, News, Foreman, Sports and wildlife. A frame is selected randomly from each video and watermarking is applied on this selected frame. Mentioned attacks are applied on the selected frames and watermark is extracted back. Peak Signal to Noise Ratio Value (between watermarked frame and original frame) and Normalized Correlation (between watermarked frame and original frame) is also represented along with attacked frame. From all the tables (Table 2 to Table 8) it is clear that with block based method of watermarking, PSNR and NC values are in the acceptable range even after attacks.

Table 9 shows the NC values between watermark image and extracted watermark image. Table 10 shows the extracted watermark after attacks.

Table 1: Watermarked Images And Extracted Watermarks from each Block with PSNR

\begin{tabular}{|c|c|c|c|c|c|}
\hline S. No. & Frame Number & PSNR & Watermarked frame & Extracted Watermark & Block for extracted watermark \\
\hline 1. & 33 & 96.314 & & $\pi \cdot 1$ & $\mathrm{Ge}\{1,1\}$ \\
\hline 2. & 66 & 96.393 & & & $\operatorname{Ge}\{1,2\}$ \\
\hline 3. & 99 & 95.992 & & & $\operatorname{Ge}\{1,3\}$ \\
\hline 4. & 132 & 96.503 & & $|Q| \mid$ & $\operatorname{Ge}\{2,1\}$ \\
\hline 5. & 165 & 96.502 & & & $\operatorname{Ge}\{2,2\}$ \\
\hline 6. & 198 & 96.374 & & MI, & $\operatorname{Ge}\{2,3\}$ \\
\hline 7. & 231 & 96.515 & & . & $\operatorname{Ge}\{3,1\}$ \\
\hline 8. & 264 & 96.464 & & ' & $\operatorname{Ge}\{3,2\}$ \\
\hline 9. & 297 & 96.350 & & "vv & $\mathrm{Ge}\{3,3\}$ \\
\hline
\end{tabular}


Table 2: Gaussian attack

\begin{tabular}{|c|c|c|c|c|}
\hline Akiyo & Carphone & Claire & Coastguard & Flower \\
\hline & & & & \\
& & & & \\
\hline PSNR - 56.75 & PSNR - 56.87 & PSNR - 56.78 & PSNR - 56.98 & PSNR - 60.86 \\
\hline NC- 0.918 & NC- 0.924 & NC- 0.926 & NC- 0.933 & NC- 0.974 \\
\hline Motherdaughter & News & Foreman & Sports & Wildlife \\
\hline PSN-56.65 & PSNR - 56.49 & PSNR - 58.91 & PSNR - 56.83 & PSNR - 57.35 \\
\hline NC- 0.931 & NC- 0.906 & NC- 0.963 & NC- 0.939 & NC- 0.951 \\
\hline
\end{tabular}

Table 3: Salt and Pepper noise

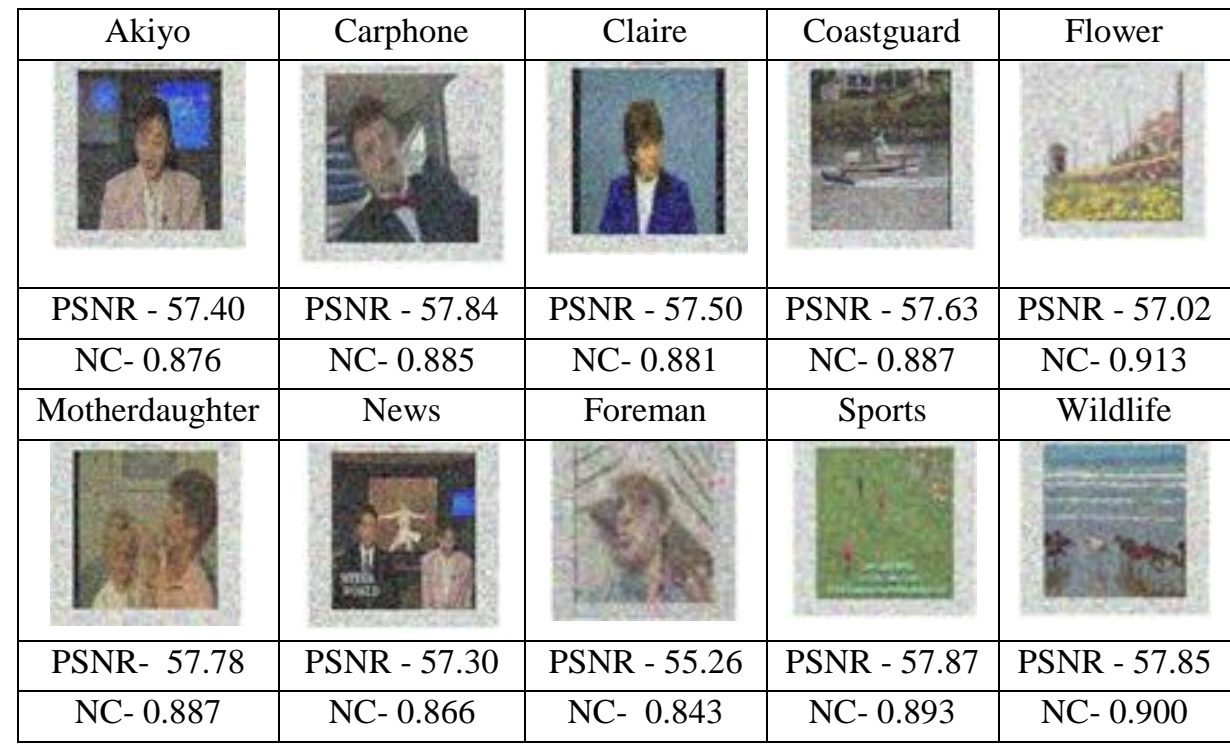

Table 4: Speckle noise

\begin{tabular}{|c|c|c|c|c|}
\hline Akiyo & Carphone & Claire & Coastguard & Flower \\
\hline & & & & \\
\hline & & & & \\
& & & & \\
\hline PSNR - 57.31 & PSNR - 57.31 & PSNR - 56.94 & PSNR - 56.92 & PSNR - 55.38 \\
\hline NC- 0.863 & NC- 0.861 & NC- 0.855 & NC- 0.857 & NC- 0.871 \\
\hline Motherdaughter & News & Foreman & Sports & Wildlife \\
\hline L & & & & \\
\hline PSNR-56.86 & PSNR - 57.62 & PSNR - 55.94 & PSNR - 56.61 & PSNR - 56.32 \\
\hline NC- 0.852 & NC- 0.864 & NC- 0.863 & NC- 0.851 & NC- 0.851 \\
\hline
\end{tabular}


Table 5: Histogram equalization

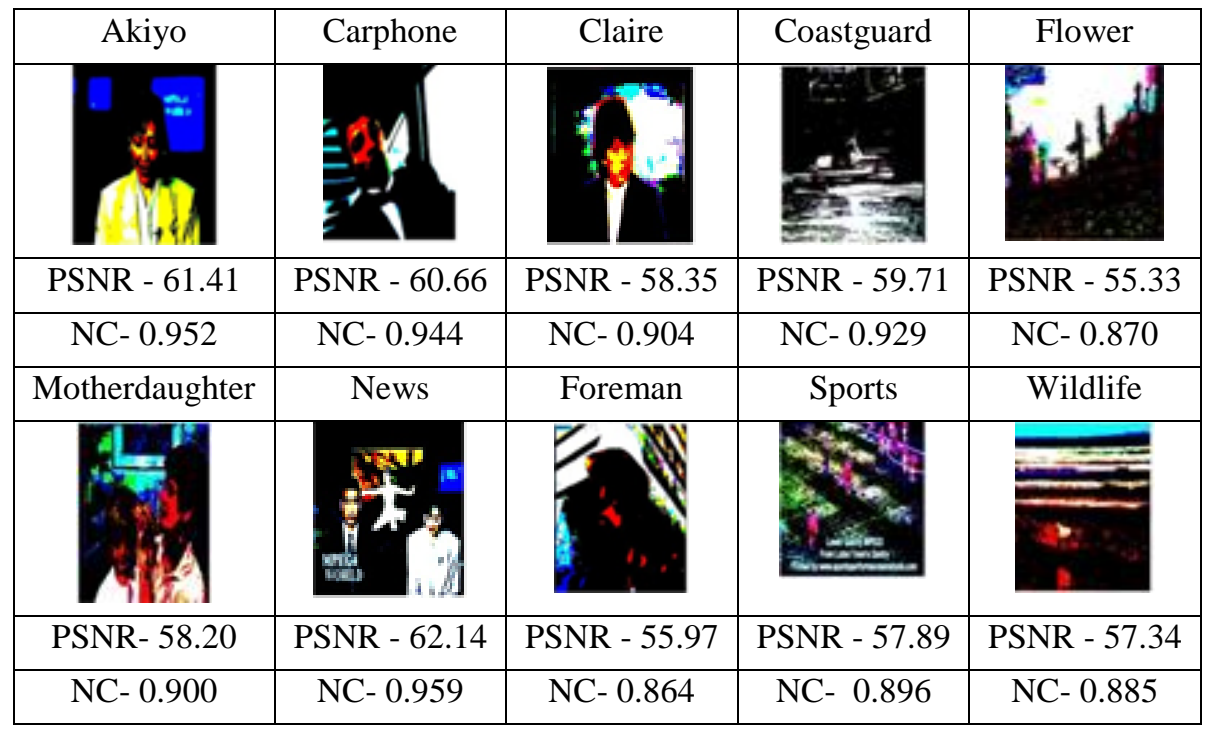

Table 6: Cropping

\begin{tabular}{|c|c|c|c|c|}
\hline Akiyo & Carphone & Claire & Coastguard & Flower \\
\hline & & & & \\
\hline PSNR - 55.91 & PSNR - 56.45 & PSNR - 55.05 & PSNR - 57.12 & PSNR - 58.13 \\
\hline NC- 0.824 & NC- 0.848 & NC- 0.779 & NC- 0.875 & NC- 0.932 \\
\hline Motherdaughter & News & Foreman & Sports & Wildlife \\
\hline & & & & \\
\hline PSNR- 58.16 & PSNR - 55.30 & PSNR - 58.36 & PSNR - 58.94 & PSNR - 59.16 \\
\hline NC- 0.890 & NC- 0.783 & NC- 0.924 & NC- 0.910 & NC- 0.924 \\
\hline
\end{tabular}

Table 7: Rotation $90^{0}$

\begin{tabular}{|c|c|c|c|c|}
\hline Akiyo & Carphone & Claire & Coastguard & Flower \\
\hline & & & & \\
\hline PSNR - 55.78 & PSNR - 56.19 & PSNR - 57.00 & PSNR - 57.15 & PSNR - 58.58 \\
\hline NC- 0.819 & NC- 0.845 & NC- 0.865 & NC- 0.874 & NC- 0.940 \\
\hline Motherdaughter & News & Foreman & Sports & Wildlife \\
\hline & & & & \\
\hline PSNR- 58.98 & PSNR - 56.39 & PSNR - 59.96 & PSNR - 60.21 & PSNR - 60.09 \\
\hline NC- 0.914 & NC- 0.832 & NC- 0.949 & NC- 0.935 & NC- 0.941 \\
\hline
\end{tabular}

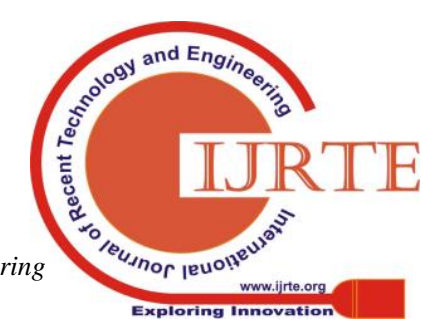


Table 8: Rotation $180^{\circ}$

\begin{tabular}{|c|c|c|c|c|}
\hline Akiyo & Carphone & Claire & Coastguard & Flower \\
\hline & & & & \\
\hline PSNR - 56.62 & PSNR - 57.51 & PSNR - 59.18 & PSNR - 56.94 & PSNR - 57.11 \\
\hline NC- 0.851 & NC- 0.886 & NC- 0.918 & NC- 0.867 & NC- 0.916 \\
\hline Motherdaughter & News & Foreman & Sports & Wildlife \\
\hline & & & & \\
\hline & & & & \\
\hline PSNR- 61.05 & PSNR - 57.48 & PSNR - 60.03 & PSNR - 62.65 & PSNR - 60.70 \\
\hline NC- 0.947 & NC- 0.869 & NC- 0.950 & NC- 0.963 & NC- 0.949 \\
\hline
\end{tabular}

Table 9: NC (Between Watermark İmage And Extracted Watermark İmage)

\begin{tabular}{|c|c|c|c|c|c|c|c|}
\hline Video Frame name & Gaussian noise & Salt pepper noise & Speckle noise & Histogram & Rotation 90 & Rotation 180 & cropping \\
\hline Akiyo & 0.851 & 0.886 & 0.888 & 0.856 & 0.854 & 0.852 & 0.851 \\
\hline Carphone & 0.853 & 0.891 & 0.885 & 0.857 & 0.854 & 0.852 & 0.851 \\
\hline Claire & 0.852 & 0.886 & 0.885 & 0.857 & 0.855 & 0.852 & 0.851 \\
\hline Coastguard & 0.851 & 0.886 & 0.893 & 0.918 & 0.852 & 0.852 & 0.851 \\
\hline Flower & 0.853 & 0.887 & 0.890 & 0.856 & 0.854 & 0.851 & 0.851 \\
\hline Foreman & 0.853 & 0.902 & 0.892 & 0.857 & 0.856 & 0.852 & 0.851 \\
\hline Mother daughter & 0.851 & 0.885 & 0.890 & 0.856 & 0.854 & 0.852 & 0.851 \\
\hline News & 0.852 & 0.887 & 0.889 & 0.855 & 0.862 & 0.852 & 0.851 \\
\hline Sports & 0.852 & 0.884 & 0.889 & 0.856 & 0.856 & 0.851 & 0.851 \\
\hline Wildlife & 0.852 & 0.882 & 0.889 & 0.857 & 0.852 & 0.852 & 0.851 \\
\hline
\end{tabular}

Table 10: Extracted Watermark after Attacks

\begin{tabular}{|c|c|c|c|c|c|c|c|}
\hline Video & Gaussian & Salt \& Pepper & speckle & $\begin{array}{c}\text { Histogram } \\
\text { Equlization }\end{array}$ & Rotation $90^{\circ}$ & Rotation $180^{\circ}$ & cropping \\
\hline Akiyo & & $\mathrm{NW}$ & "..WV & ו'ו ו'י & $1 \%$ & I. & \\
\hline Carphone & & 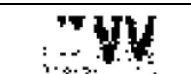 & " WV & & & & \\
\hline Claire & TT & $\because \mathrm{WV}$ & $" \sqrt{W}$ & & & & \\
\hline Coastguard & & WV & $\mathrm{HW}$ & & & & \\
\hline Flower & & . WW & $\therefore \mathrm{WW}$ & 17 & & & \\
\hline Foreman & & "HU & $\therefore \mathrm{HW}$ & & I & "VW & \\
\hline Motherdaughter & 17 & " & $\because \mathrm{WV}$ & & 17 & 해 & \\
\hline News & 17 & $\because \mathrm{WV}$ & $\because \mathrm{WV}$ & 17 & & & \\
\hline Sports & בוד & WU & $\mathrm{WU}$ & 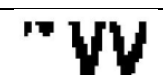 & WW & & \\
\hline Wildlife & WV & $\therefore \mathrm{WU}$ & $\therefore \mathrm{HW}$ & & 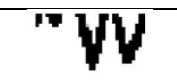 & F Wh & ${ }^{2}$ \\
\hline
\end{tabular}

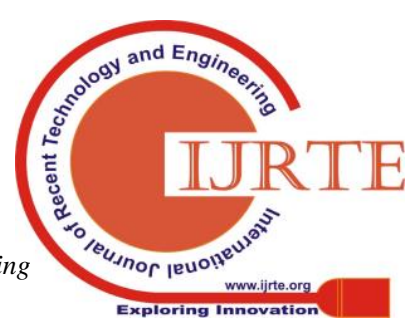




\section{COMPARISION OF PROPOSED SCHEME WITH EXISTING SCHEMES}

The novelty of this paper can be represented and discussed. DWT and SVD methods are used here to gain the advantages of both transforms. To obtain better performance in terms of imperceptibility, block based method is used and the watermark is embedded in only one block of every selected frame.

This enhances the security of the watermarking scheme as complete watermark can not be extracted from the same frame of the video. So, any attacker needs to know all the frames and the block in each frame used for embedding watermark. A comparision of proposed method with previously existing methods is shown in Table 11. The calculated PSNR of proposed method is much better than ( Adul \& Mwangi, 2017)and(Panyavaraporn \& Paramate, 2018). It is also shown here through bar graph.

Table 11: Comparison of Proposed Method with Existing Methods

\begin{tabular}{|c|c|c|c|c|c|}
\hline $\begin{array}{c}\text { S.N } \\
0 .\end{array}$ & $\begin{array}{c}\text { Method } \\
\mathrm{s}\end{array}$ & videos & Frames & $\begin{array}{c}\text { Extracted } \\
\text { Watema } \\
\mathrm{rk}\end{array}$ & PSNR \\
\hline 1. & $\begin{array}{c}\text { Propos } \\
\text { ed } \\
\text { Method }\end{array}$ & Akiyo & & & \\
\hline 2. & $\begin{array}{c}\text { Ref } \\
{[19]}\end{array}$ & Foreman & & 96.393 \\
\hline 3. & $\begin{array}{c}\text { Ref } \\
{[20]}\end{array}$ & $\begin{array}{c}\text { Basketb } \\
\text { all }\end{array}$ & $\mathbf{4}$ & 36.6 \\
\hline
\end{tabular}

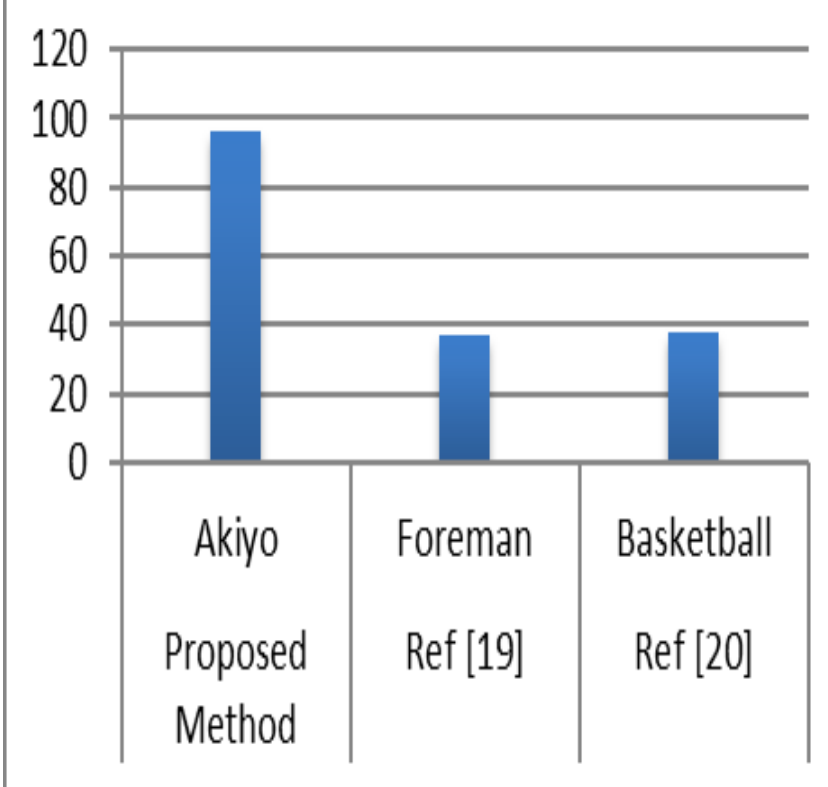

Fig. 4: Comparison graph of Three video framesProposed- Akiyo, Ref. [19]- Foreman, Ref. [20]Basketball

\section{CONCLUSION}

In this paper effect of attacks after block based watermarking is shown. In this method, different part of watermark are embedded in different frames of video hence, to completely extract the watermark one should know the exact frames in which watermark is embedded and again the part of that frames in which watermark has been embedded. This scheme gives improved PSNR and improved security. Also unlike many other schemes watermark is not embedded in every frame of the video, so the time required for watermarking is less.

\section{REFERENCES}

1. Adul , V., \& Mwangi, E. (2017, Sept.). A Robust Video Watermarking Approach based on a hybrid SVD/DWT Technique. IEEEAfricon.

2. Lu , C., \& Liao, H. (2001, Oct.). Multipurpose watermarking for image authentication and protection. IEEE Transactions on Image Processing,, 1579-1592.

3. Lu, C., Huang, S., Sze, C., \& Lia, H. (2000, Dec.). Cocktail watermarking for digital image protection. IEEE Transactions on Multimedia, 2(4), 209-224.

4. Stelter, ]., \& Stone, B. (Feb. 2009.). Digital pirates winning battle with studios . The New York Times,

5. Allali, A., Elabbadi, J., \& Elhassa. (2012). Video object watermarking using 3D-Walsh Hadamard transform and Arnold transform. IEEE International Conference on Multimedia Computing and Systems (ICMCS), (pp. 119 - 124).

6. Asikuzzaman, M., \& Pickering, M. R. (2017). An overview of digital video watermarking. IEEE transactions on circuits and systems for video technology, 28(9), 2131-2153.

7. (2011). Economic consequences of movie piracy Australia . The media, content and technology research specialist.

8. Hsiao, J., Chen , C., Chien, L., \& Chen. (2007). A new approach to image copy detection based on extended feature sets,. IEEE Transaction on Image Processing, 16(8), 2069-2079.

9. Khan, A., Tahir, S. F., Majid, A., \& Choi, T. S. (2008) Machine learning based adaptive watermark decoding in view of anticipated attack. Pattern Recognition, 41(8), 2594-2610.

10. Koch, J., Smith, M., \& Telang, R. (2011). Camcording and film piracy in asia-pacific economic cooperation economies. International Intellectual Property Institute.

11. Kong, W., Yang, B., Wu, D., \& Niu, X. (2006). SVD based blind video watermarking algorithm. IEEE First International Conference on Innovative Computing , Information and Control(ICICI), 1, pp. 1-4. Beijing, China,.

12. Kutter, M., Voloshynovskiy, S., \& Herrigel , A. (2000). Watermark copy attack. Proceedings of Security and Watermarking of Multimedia Contents II,, 3971. San Jose, CA, United States.

13. Panyavaraporn, J., \& Paramate, H. (2018). DWT/DCTbased Invisible Digital Watermarking Scheme for Video Stream. IEEE 10th International Conference on Knowledge and Smart Technology (KST).

14. Peng, H., Wang, J., \& Wang, W. (2010). Image watermarking method in multiwavelet domain based on support vector machines. The Journal of Systems and Software, 83(8), 1470-1477.

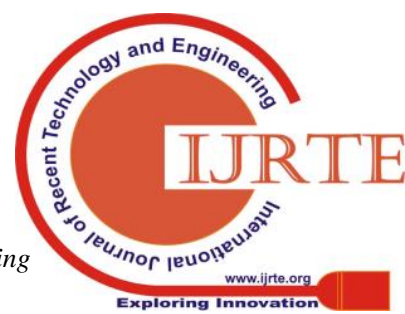


15. Piva, A., Bartolini, F., \& Barni, M. (2002, May). Managing copyright in open networks. IEEE Internet Computing, 6(3), 18-26.

16. Rao, T., \& Rao, K. (2016). Integrity based video watermarking using gaussian based dwt embedding and extraction process., , pp. 3235-3240. International Journal of Applied Engineering Research, 11(5).

17. Sharma , V., \& Mann, P. (2017, March). A Review on Various Optimized Image Watermarking Techniques. International Journal of Computer Applications, 162(2), 26-30.

18. Thind, D., \& Jindal, S. (2015). A Semi Blind DWT-SVD Video Watermarking . International Conference on Information and Communication Technologies, (pp. $1661-1667)$.

19. Tsai, J., Huang, W., \& Kuo, Y. (2011, Mar.). On the selection of optimal feature region set for robust digital image watermarking. IEEE Transactions on Image Processing, 20(3), 735-743.

20. Wenhai Kong, B. Y., Wu, D., \& X. N. (2006). SVD Based Blind Video Watermarking Algorithm. IEEE Proceedings of the First International Conference on Innovative Computing, Information and Control, (pp. 1$4)$. 